\title{
Ensemble Kalman Filter Finite Element Method Applied to Dynamic Motion of Elastic Body
}

\author{
Yukiko TEZUKA $^{1}$ and Mutsuto KAWAHARA ${ }^{2}$ \\ ${ }^{1}$ Department of Civil Engineering, Chuo University, Japan (yuki-tez@ civil.chuo-u.ac.jp) \\ ${ }^{2}$ Department of Civil Engineering, Chuo University, Japan (kawa@ civil.chuo-u.ac.jp)
}

\begin{abstract}
The purpose of this research is to obtain an estimation of behavior of an elastic body based on errorneous observations. To do this, the ensemble Kalman filter finite element method is used. In numerical study, a three dimensional elastic body is considered. The Iwatayama tunnel is employed. This construction site is located in Gifu prefecture, in Japan. The external force is applied to the tunnel face. As the boundary condition, bottom of the computational model is fixed in all directions. Dynamic motion is caused by the external force applied to the body. Observation data of acceleration at observation points are necessity to estimate acceleration at some other points. In this study, artificial data is used. Computed values plus the white Gaussian noise is used as artificial observations. The finite element method is applied to the spatial discretization and the linear acceleration method(Newmark $\beta$ method) is used as the temporal interpolation. Finally, the estimation is compared with the results by the finite element method, and effectiveness of the EnKF is verified. We suggest that an ensemble of size 40 and 100 to consider the relation between the size of ensemble members and accuracy of the EnKF.
\end{abstract}

Keywords: Finite element method, Ensemble Kalman filter, Iwatayama tunnel.

\section{INTRODUCTION}

The measurements of the blasting vibration in tunnel excavation are often carried out. However, natural phenomena generally consist of uncertain values and include several noises. In recent years, computer technology made tremendous progress and numerical analysis is effective in estimating natural phenomena.In general, the finite element method is applied to the numerical simulation in the field of geomechanics.

To get fintered results, the Kalman filter (KF) method is useful, because the Kalman filter can filter artificial and mechanical errors. It is useful for all engineering fields. The problem is that Kalman filter method is capable to estimate only in the time direction. To overcome this problem Kalman filter will be combined with the finite element method (KF-FEM). By this method, state values can be estimated in the time and space directions. However we 
need huge computational requirements, to overcome this difficulty the ensemble Kalman filter $(\mathrm{EnKF})$ is created. The state values sare highly uncertain and an infinite number of samples are nesessary. In nature, this is not really the case: there is geological uncertainty about different loading conditions, for example. One way to deal with this uncertainty is the random finite element method. Several simulations are carried out to give an ensemble of state value which are needed in EnKF. Parameters can be estimated using the limited number of observation data by combining the EnKF with the random finite element method. The present study investigates whether the EnKF finite element method can be adaptable to the dynamic motion of a three dimensional elastic body.By applying the estimation technique at the Iwatayama tunnel site, we verify that the method is useful for tunnel excavation.

\section{FINITE ELEMENT METHOD}

\subsection{Basic Equation and Boundary Condition}

In this study, indicial notation and the summation convention are used to describe equation. Eqs.(1) (3) are the basic equations of the elastic body.

$\langle$ Balance of stress equation $\rangle$

$$
\sigma_{i j, j}-\rho \ddot{u}_{i}=0
$$

$\langle$ Strain displacement equation〉

$$
\varepsilon_{i j}=\frac{1}{2}\left(u_{i, j}+u_{j, i}\right)
$$

$\langle$ Stress-strain equation $\rangle$

$$
\sigma_{i j}=D_{i j k l} \varepsilon_{k l}
$$

where $\sigma_{i j}, \rho$ and $\ddot{u}_{i}$ are overall stress, density and acceleration, respectively. $D_{i j k l}$ is called as the elastic coefficient matrix.

\section{THE ENSEMBLE KALMAN FILTER}

In the conventional Kalman filter, huge computational load is necessary to find the inverse of the matrix. In addition, the errors make prediction of estimation worse. To overcome these difficulties the ensemble Kalman filter is originated by Evensen(1994). An ensemble of possible state vectors are considered, which are generated using the random finite element method, to represent statistical spread of the state vector. The ensemble Kalman filter consists of three steps. The first step is the forecast step: To represent the errorneous behavior, we assume that at time $k$, an ensemble of $q$ forecasted state $x_{k}^{f_{q}}$ estimates random simple error statistics. This ensemble is denoted by $X_{k}^{f} \in R^{n \times q}$, where

$$
X_{n}^{f} \equiv\left(x_{n}^{f_{1}}, \cdots, x_{n}^{f_{q}}\right) .
$$

The ensemble mean $\bar{x}_{n}^{f} \in R^{n}$ is defined by

$$
\bar{x}_{n}^{f} \equiv \frac{1}{q} \sum_{i=1}^{q} x_{n}^{f_{i}} .
$$


An ensemble error matrix $C_{n}^{f} \in R^{n \times q}$ is expressed as follows.

$$
C_{n}^{f} \equiv\left[x_{n}^{f_{1}}-\bar{x}_{n}^{f}, \cdots, x_{n}^{f_{q}}-\bar{x}_{n}^{f}\right]
$$

The second step is the analysis step: To obtain the estimate of the state, the EnKF performs an ensemble of the parallel data assimilation cycles, for $i=1, \cdots, q$

$$
x_{n}^{a_{i}}=x_{n}^{f_{i}}+\hat{K}_{n}\left(y_{n}^{f_{i}}-h\left(x_{n}^{f_{i}}\right)\right) .
$$

The perturbed observations $y_{n}^{i}$ are assumed as;

$$
y_{n}^{i}=y_{n}+v_{n}^{i} .
$$

Thinking of the forecasted ensemble mean as the best estimate of the state, and spread of the ensemble members as the error between best estimate and true state, we approximate the analysis error covariance as follows;

$$
\hat{P}_{n}^{a} \equiv \frac{1}{q-1} C_{n}^{a}\left(C_{n}^{a}\right)^{T}
$$

where $C_{n}^{a}$ is defined by Eq.(??), and $x_{n}^{f_{i}}$ is replaced by the mean of the analysis estimate ensemble members. This is different from Eq.(??), so a term is added.

$$
\hat{P}_{n}^{a}=\left(I-K_{n} H\right) C_{n}^{f} .
$$

The Kalman gain is obtained by the approximation of the error covariance as,

$$
\hat{K}_{n}=C_{n}^{f}\left[(q-1) I+\left(H C_{n}^{f}\right)^{T} R^{-1} H C_{n}^{f}\right]^{-1}\left(H C_{n}^{f}\right) R^{-1} .
$$

In the EnKF, the Kalman gain can be obtained using the square matrix of order $q$.

Ensemble members is updated,and ensemble mean is replaced by the mean of the analysis estimate ensemble members. Estimation value is calculated.

$$
\bar{x}_{n}^{a}=\frac{1}{q} \sum_{i=1}^{q} x_{n}^{a_{i}} .
$$

The last step is to obtain the prediction of error statistics using the values of the forecast step:

$$
x_{n+1}^{f_{i}}=f\left(x_{n}^{a_{i}}, u_{n}\right)+w_{n}^{i} .
$$

For the elastic vibration equation, the discretized by the finite element method is used.

\section{NUMERICAL STUDY}

\subsection{A Three Dimensional Elastic Body:Iwatayama Tunnel}

A construction site of Iwatayama tunnel which is located in Gifu prefecture, Japan, and employed for the practical site. The mountain is close to the residential area, and has steep rock slope. Therefore it is important to make a close examination of rock vibration. 


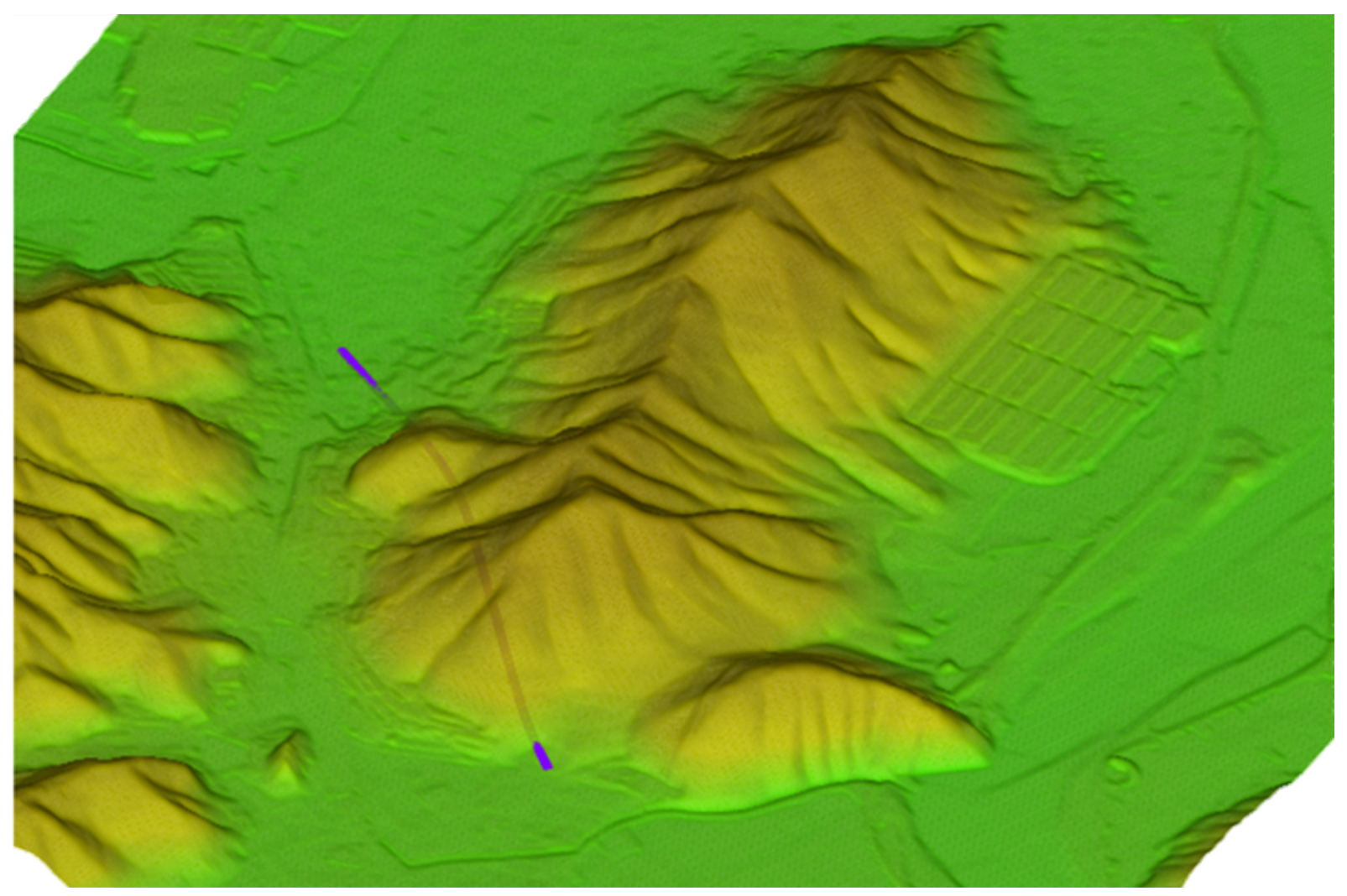

Figure 1. digital map of the Iwatayama tunnel

The digital map of Iwatayama tunnel is shown in Figure 1. The finite element mesh used is shown in Figure 2. The total number of nodes and elements are 3746 and 18678 , respectively. The size of the body is $200 \times 200 \times 110[\mathrm{~m}]$. As a boundary condition, bottom is fixed, and the other surface is free. Poisson ratio and density are set as 0.30 and $2.0 \times$ $10^{3}\left[\mathrm{~kg} / \mathrm{m}^{3}\right]$, respectively. Each element is assumed to have a random elastic modulus. In this study damping coefficient $\alpha_{0}$ and $\alpha_{1}$ are set as $0.001[1 / \mathrm{sec}]$ and $0.01[\mathrm{~m} / \mathrm{sec}]$. 


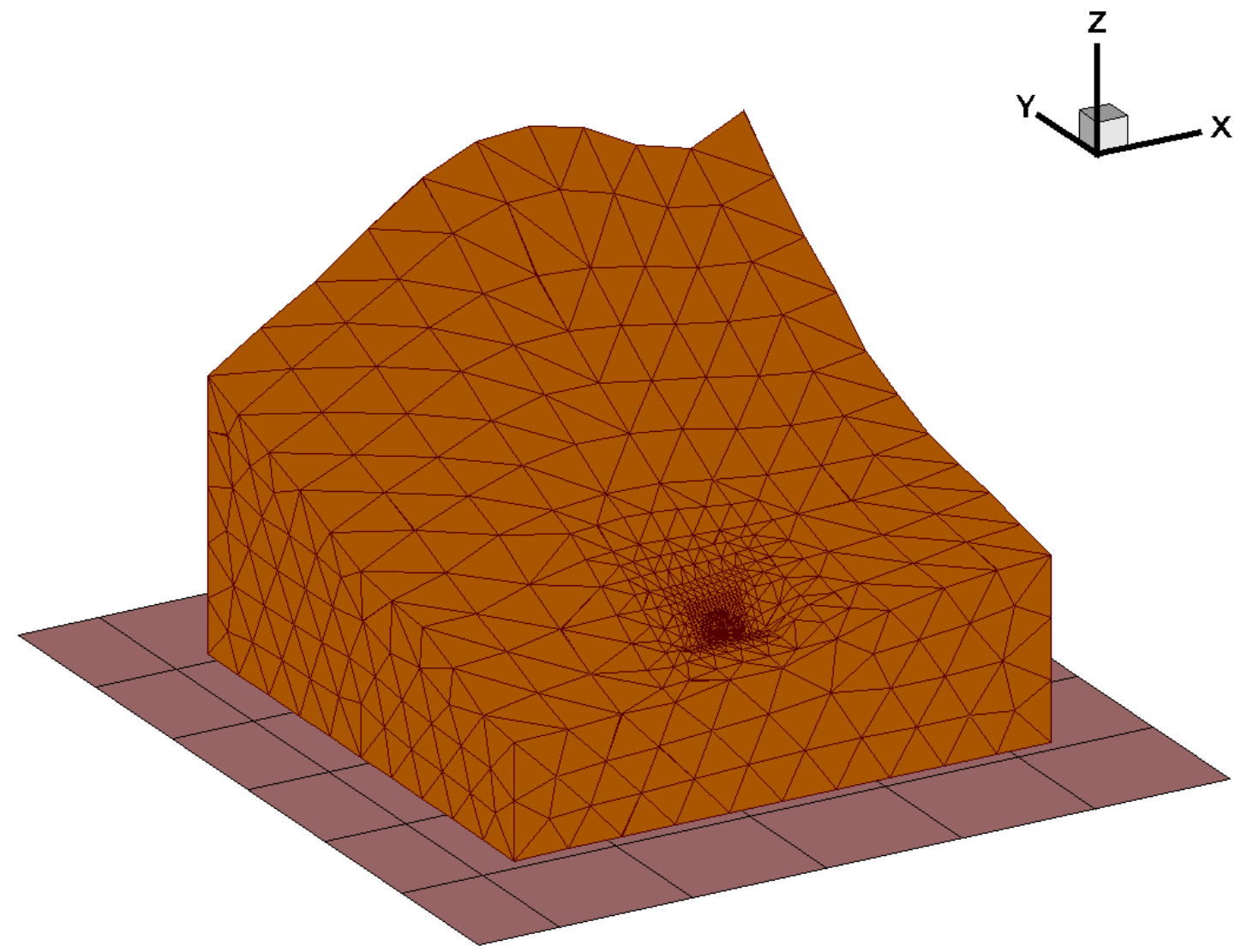

Figure 2. a finite element mesh

The domain is assumed to be subjected to the external force at the tunnel face, which is expressed by

$$
\hat{\Gamma}_{\alpha i}=\int_{\Gamma} A_{\alpha i}\left(e^{-\xi t}-e^{-\eta t}\right) d S .
$$

where $A_{\alpha i}$ is the magnitude of external force. The mesh is assumed that the tunnel was constructed about $70[\mathrm{~m}]$ from tunnel mouth as shown in Figure 3. Figure 3 shows the schematic view of the tunnel face. 


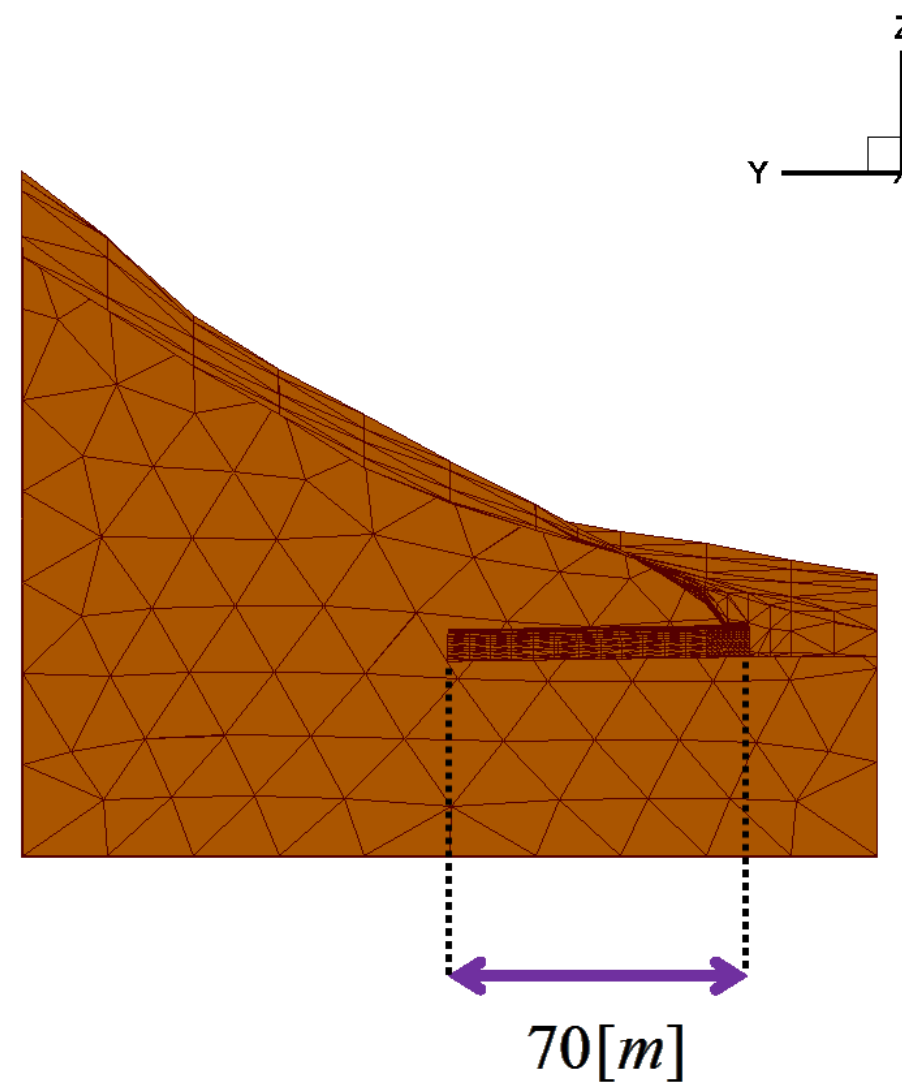

Figure 3. Cross section

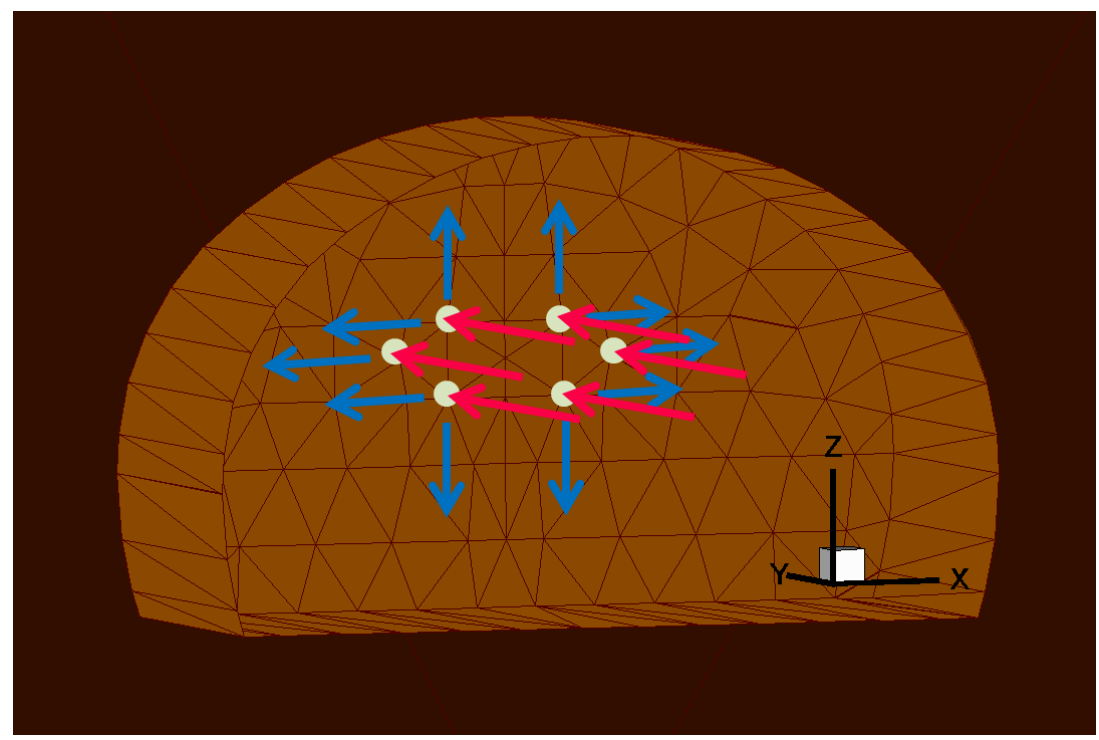

Figure 4. Tunnel face

Observation and estimation points are set as shown in Figure 5. Acceleration at estimation point is estimated using the observation data. This data is shown in the next section. 


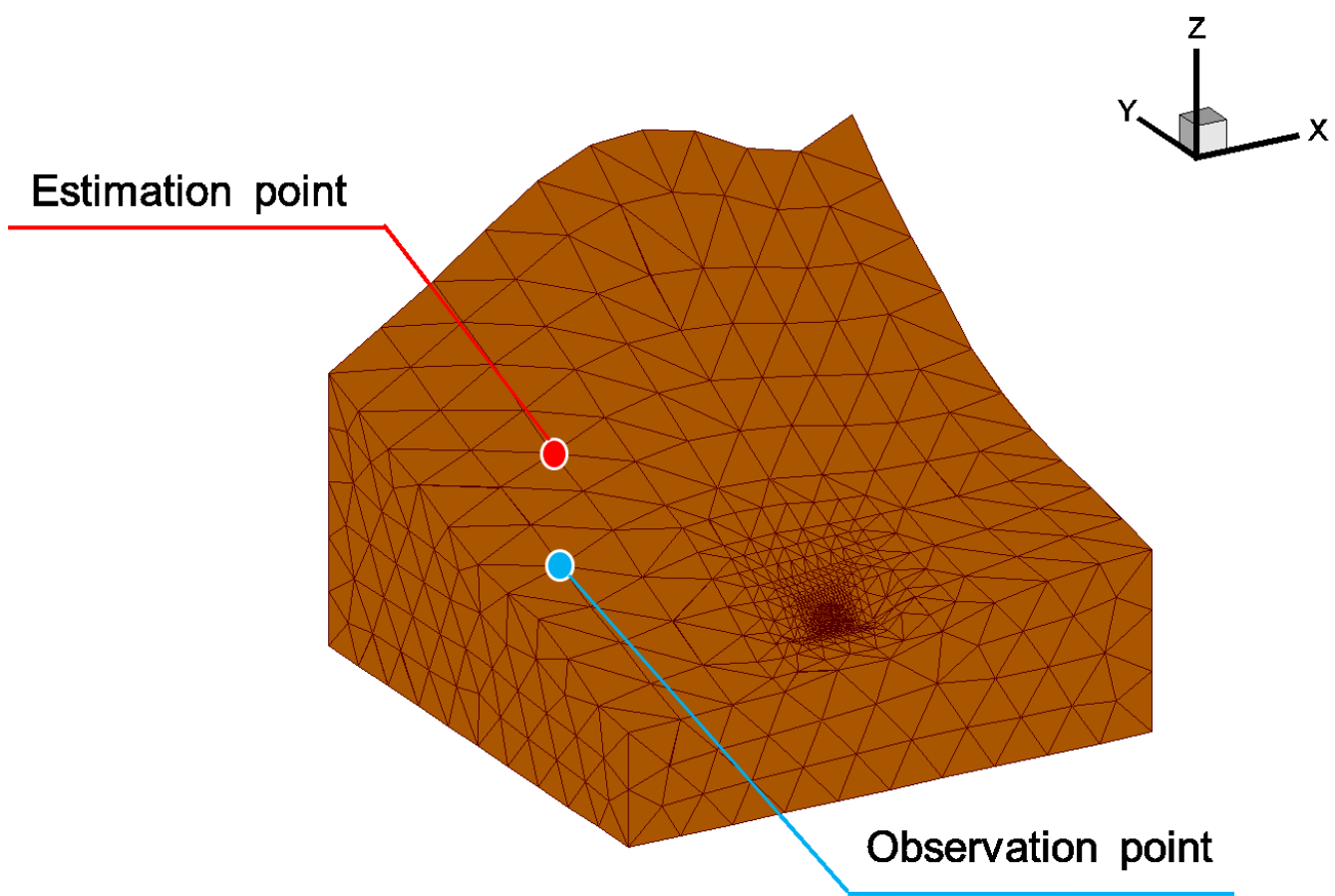

Figure 5. observation point and estimation point

The random finite element method combines the finite element method with the random field theory. In this study, the elastic modulus and magnitude of the external force are randomized. Elastic modulus based on a standard distribution can be transformed into those on a normal distribution. For each element:

$$
E_{i}=\mu_{E}+\sigma_{E} Z_{i}
$$

where $\mu_{E}$ is mean of the elastic modulus, and $\sigma_{E}=0.01$ is standard deviation of the elastic modulus, and $i$ means number of element. $\mu_{E}$ is set $1.0 \times 10^{9}\left[\mathrm{kN} / \mathrm{m}^{3}\right]$ in this study.

$$
A_{\alpha i}=\mu_{A_{\alpha i}}+\sigma_{A_{\alpha i}} Z_{i}
$$

where $\mu_{A_{\alpha i}}$ is mean of magnitude of the external force, and $\sigma_{A_{\alpha i}}=0.01$ is the standard deviation of the magnitude. $\mu_{A^{i}}$ is set $1.0 \times 10^{8}\left[\mathrm{kN} / \mathrm{m}^{3}\right]$.

The random variable based on standard normal distribution is represented by $Z_{i}$.

\subsection{Observation Data and Ensemble Members}

Observation data are obtained by the random finite element method. 100 ensemble members are used at each time step. In these figures, a solid line is ensemble mean. The distribution of the ensemble is shown in Figures 6, 7 and 8. These figures show values on $\mathrm{X}, \mathrm{Y}, \mathrm{Z}$-direction, respectively. 


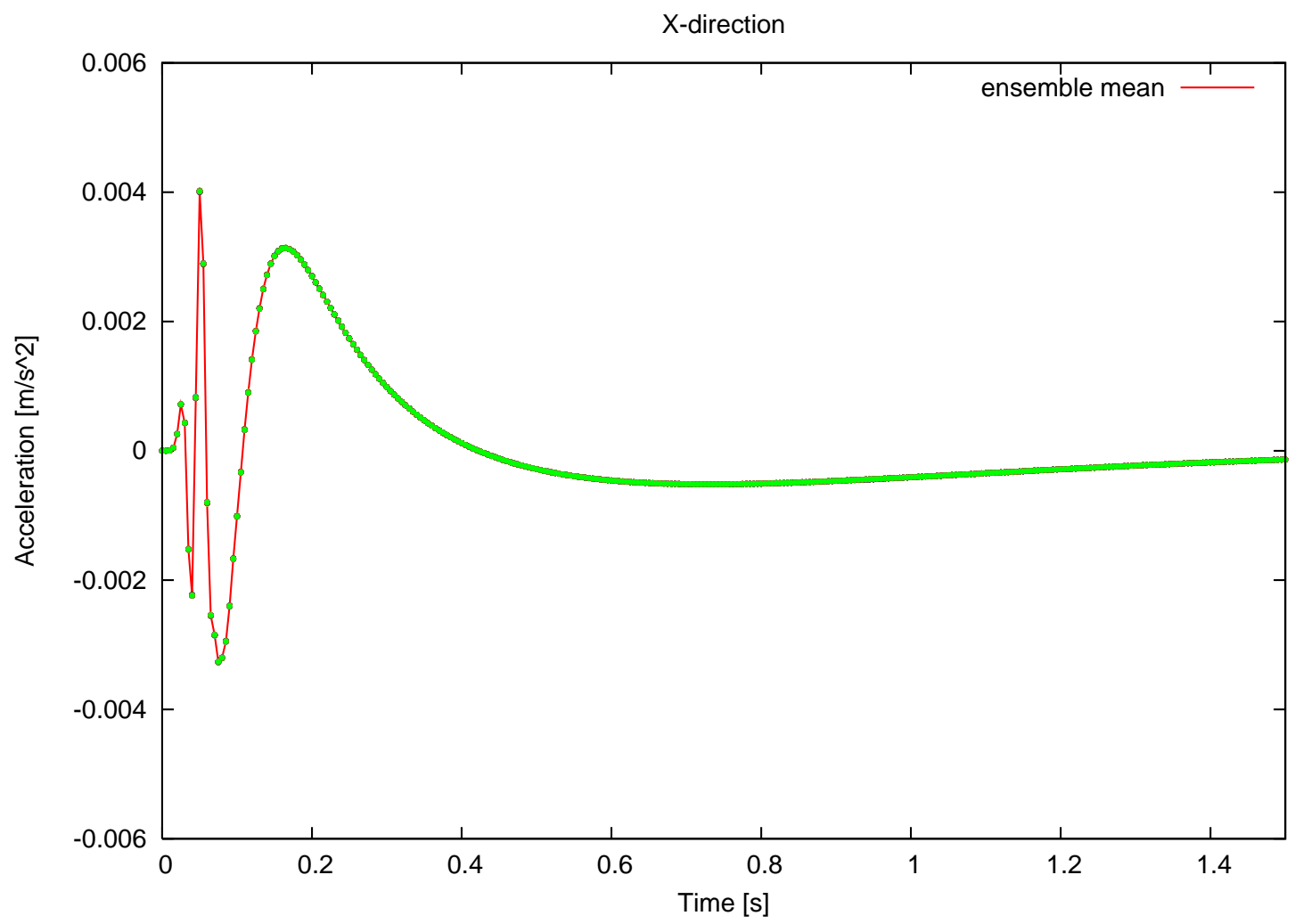

Figure 6. the ensemble at $\mathrm{X}$-direction

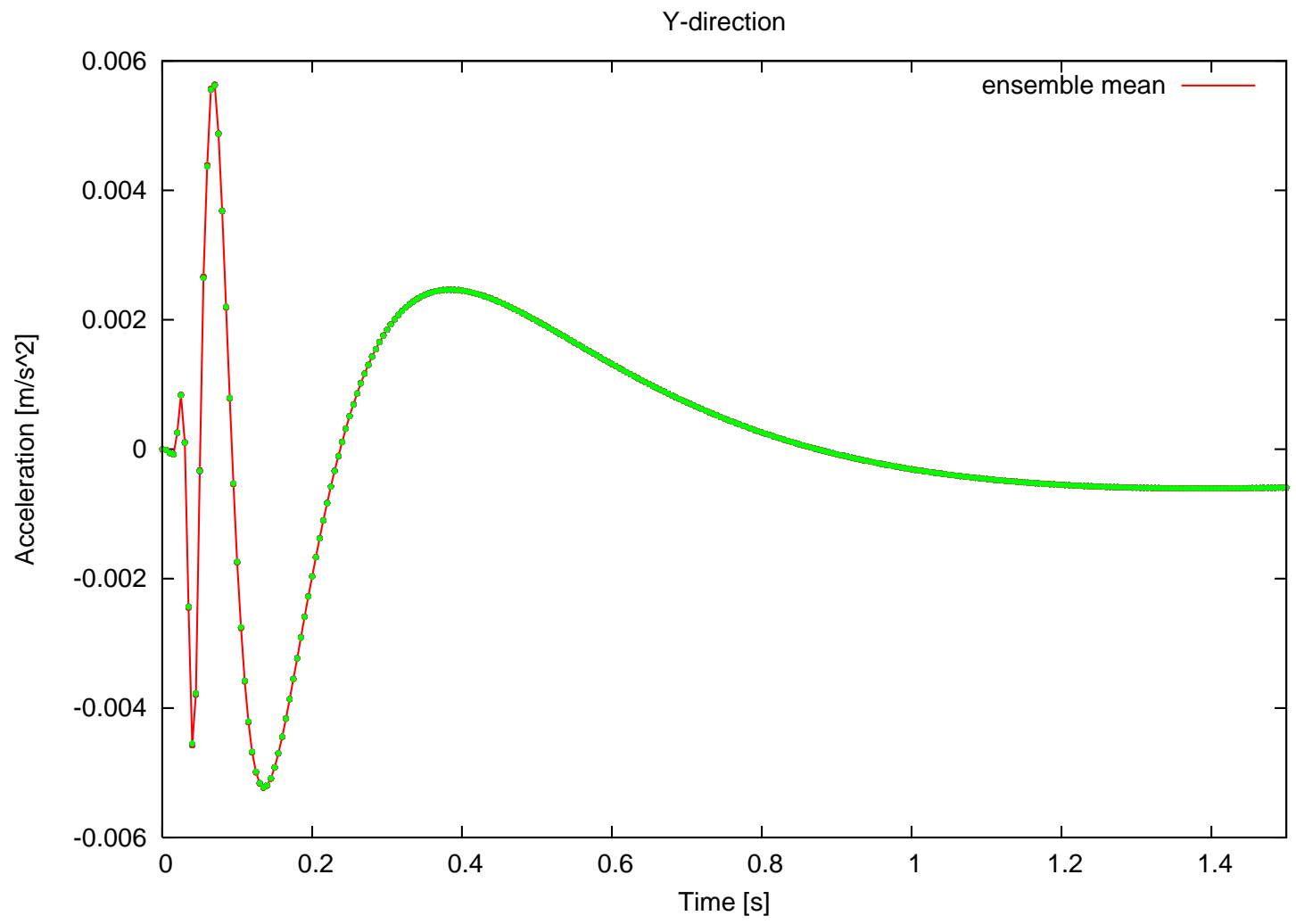

Figure 7. the ensemble at Y-direction 


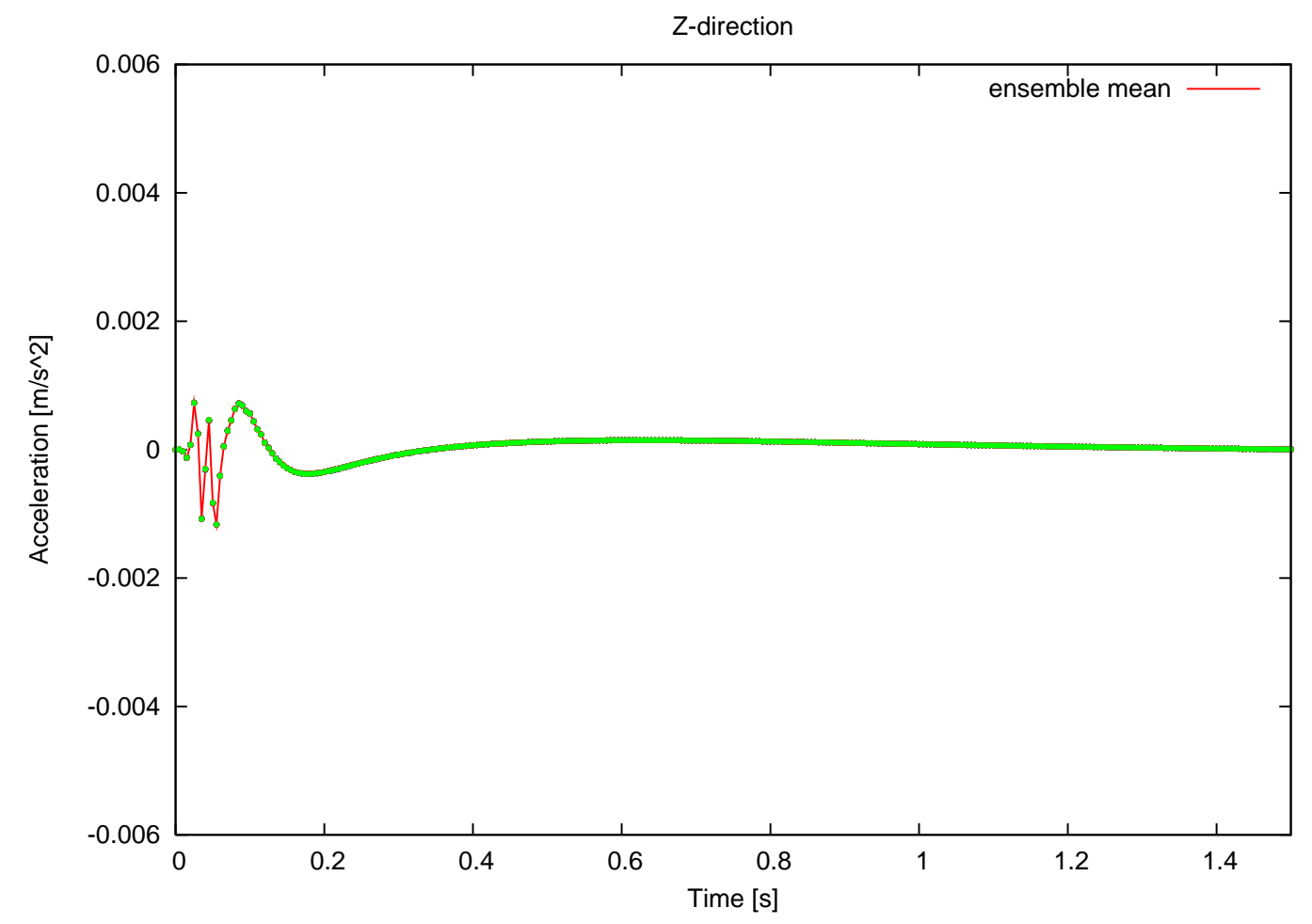

Figure 8. the ensemble at Z-direction

\subsection{Verification}

Figures 12, 13 and 14 show the comparisons of acceleration between estimation value using the EnKF finite element method and artificial observation value using the finite element method. The estimation values can be found between the range of the ensemble members. The filtering performed well. 


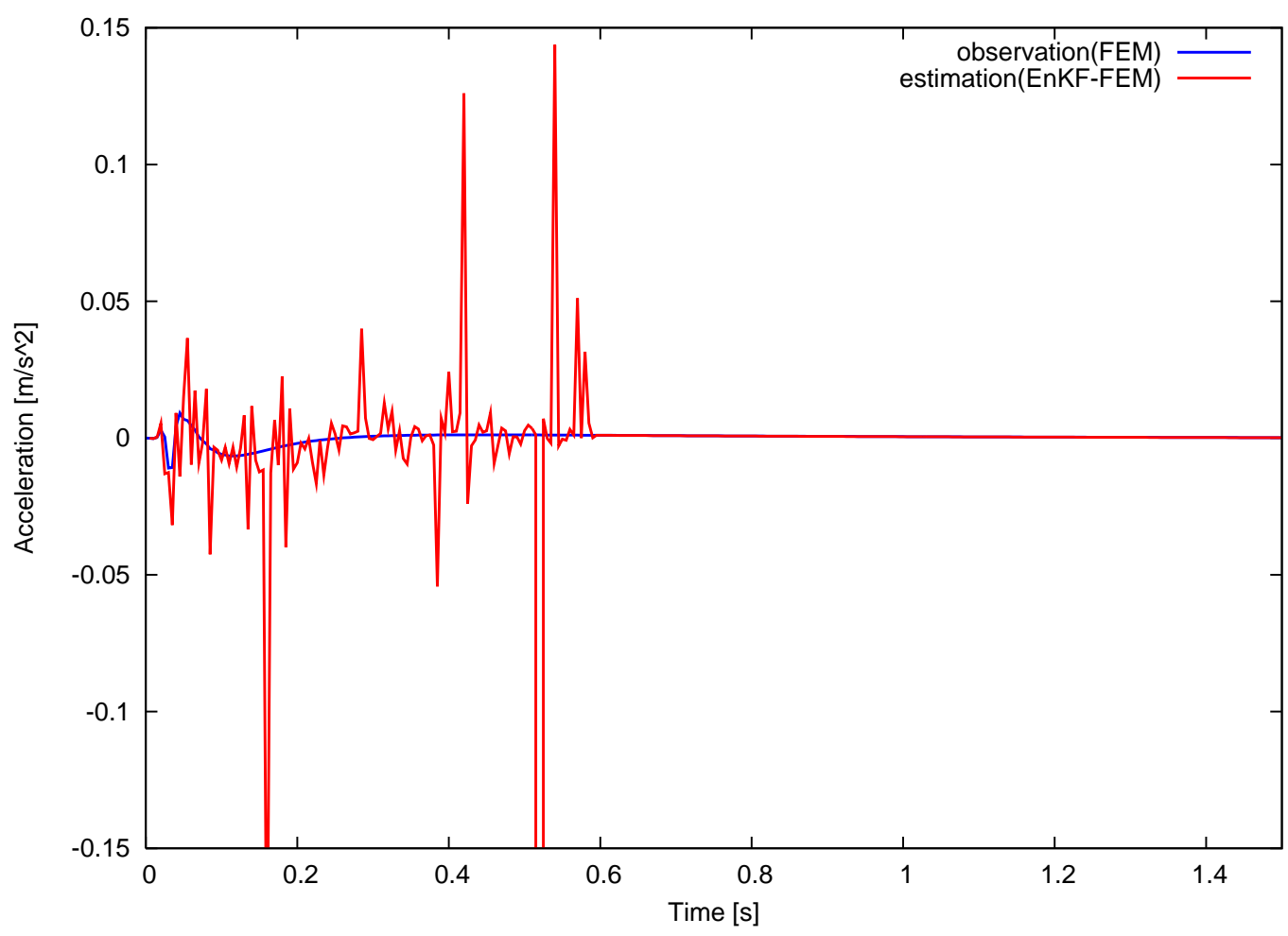

Figure 9. X-acceleration at estimation point

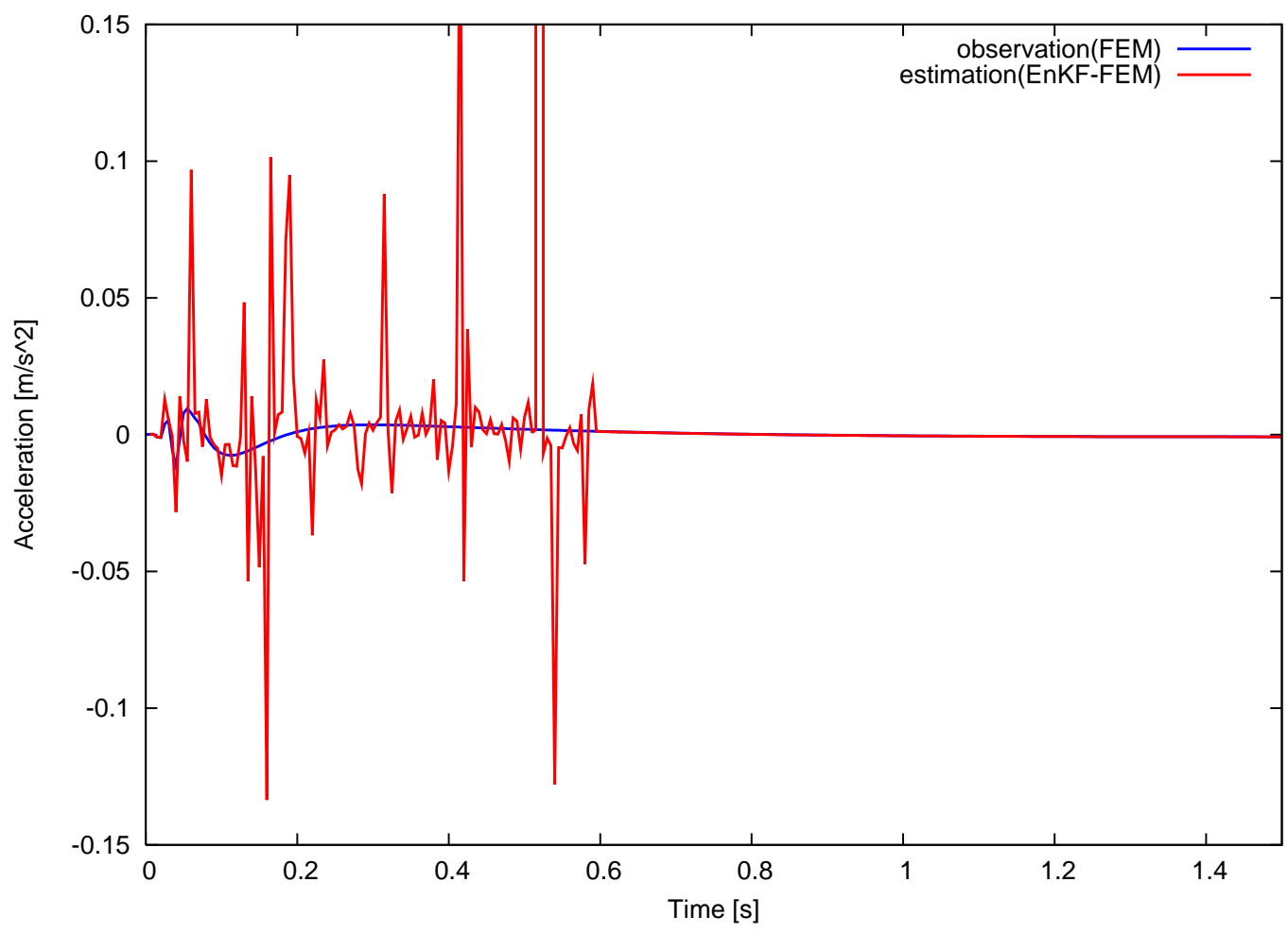

Figure 10. Y-acceleration at estimation point 


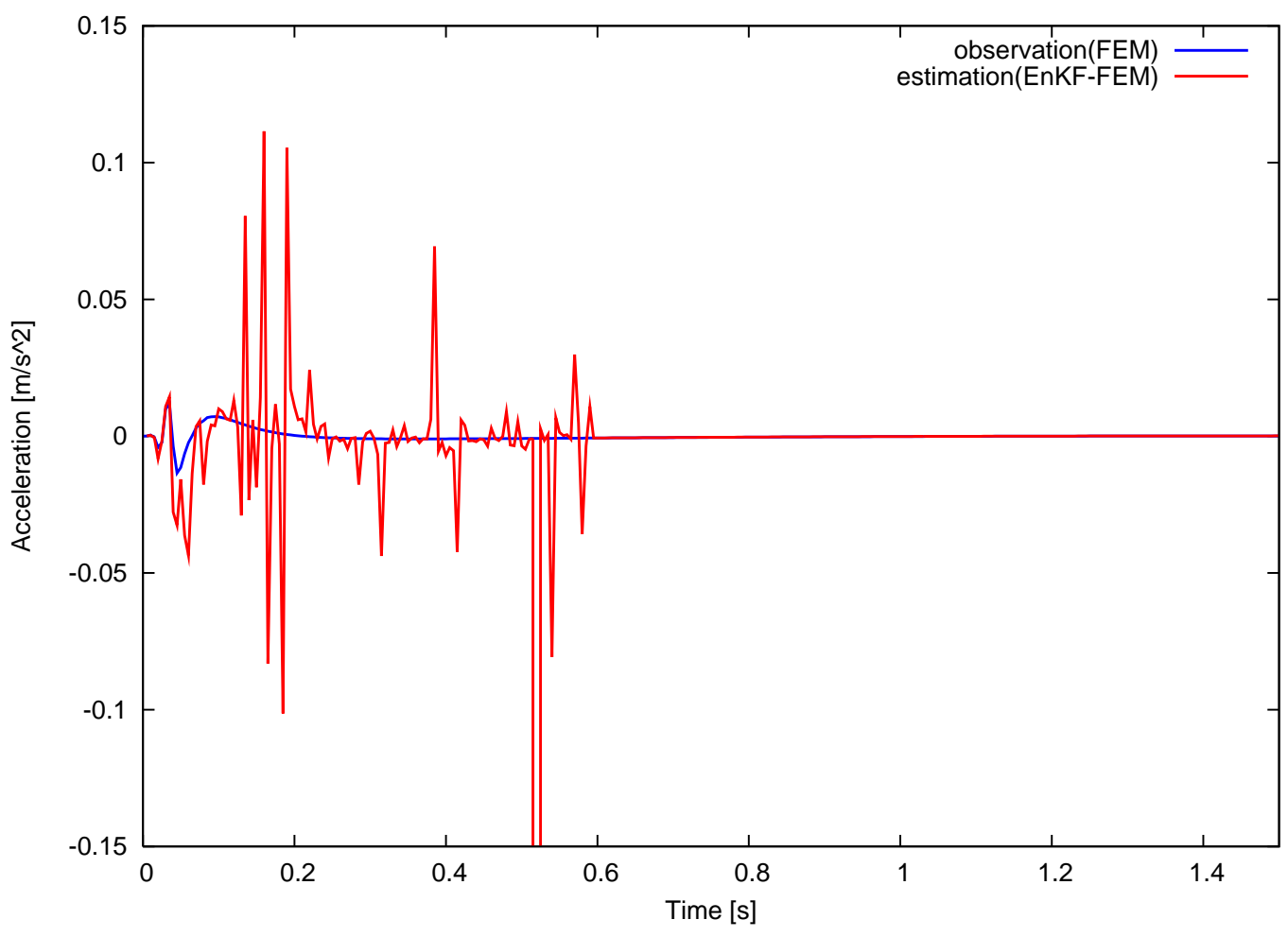

Figure 11. Z-acceleration at estimation point

\subsection{Comparison}

We start with comparing the size of the ensemble members. We make comparison between results with 40 ensemble members and 100 ensemble members. A low number ensemble members make the range of error large.In contrast, if the number of ensemble members increase, accuracy of the EnKF is improved. It is seen that the estimated acceleration with 40 ensemble members is almost coincident with the observed acceleration. 


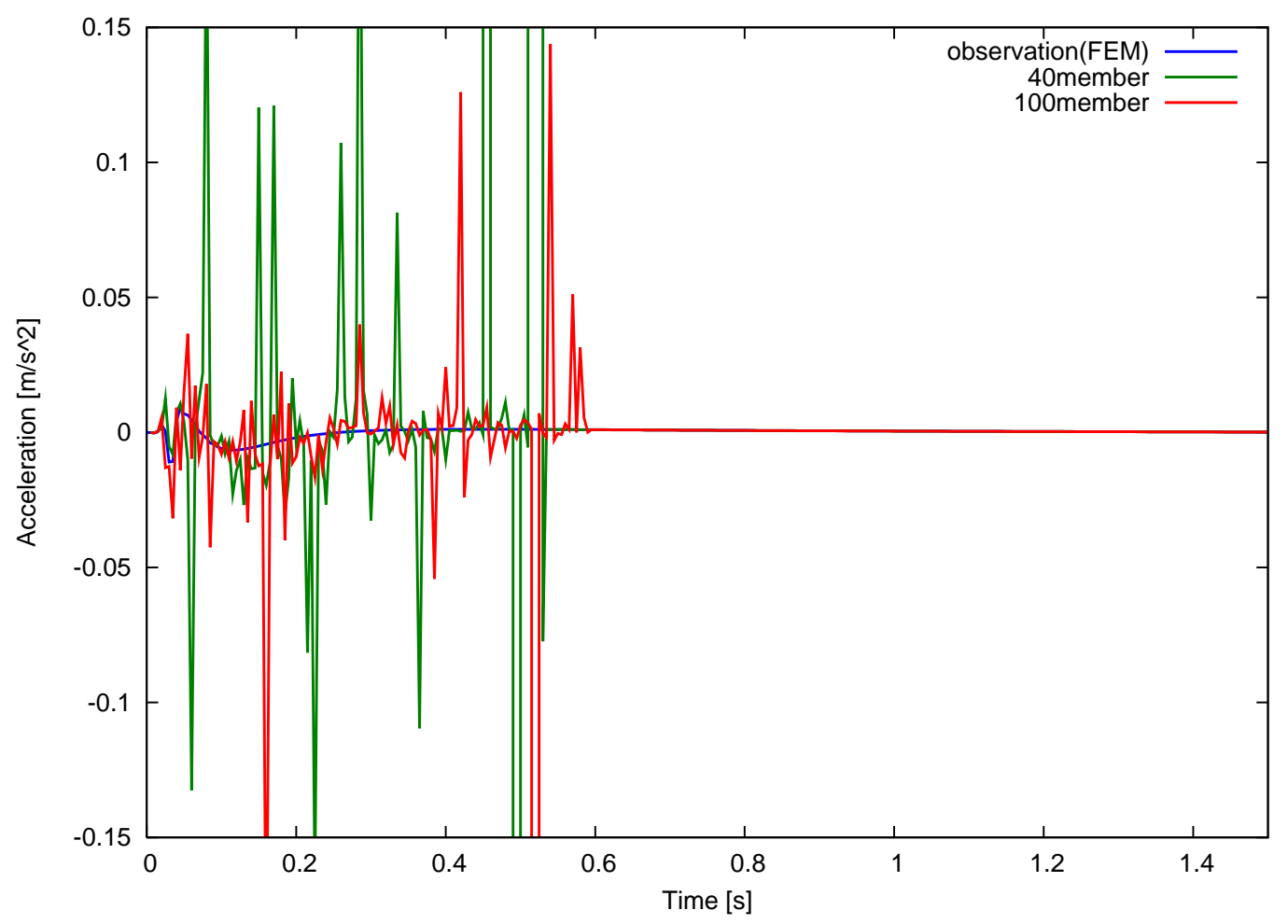

Figure 12. X-acceleration at estimation point

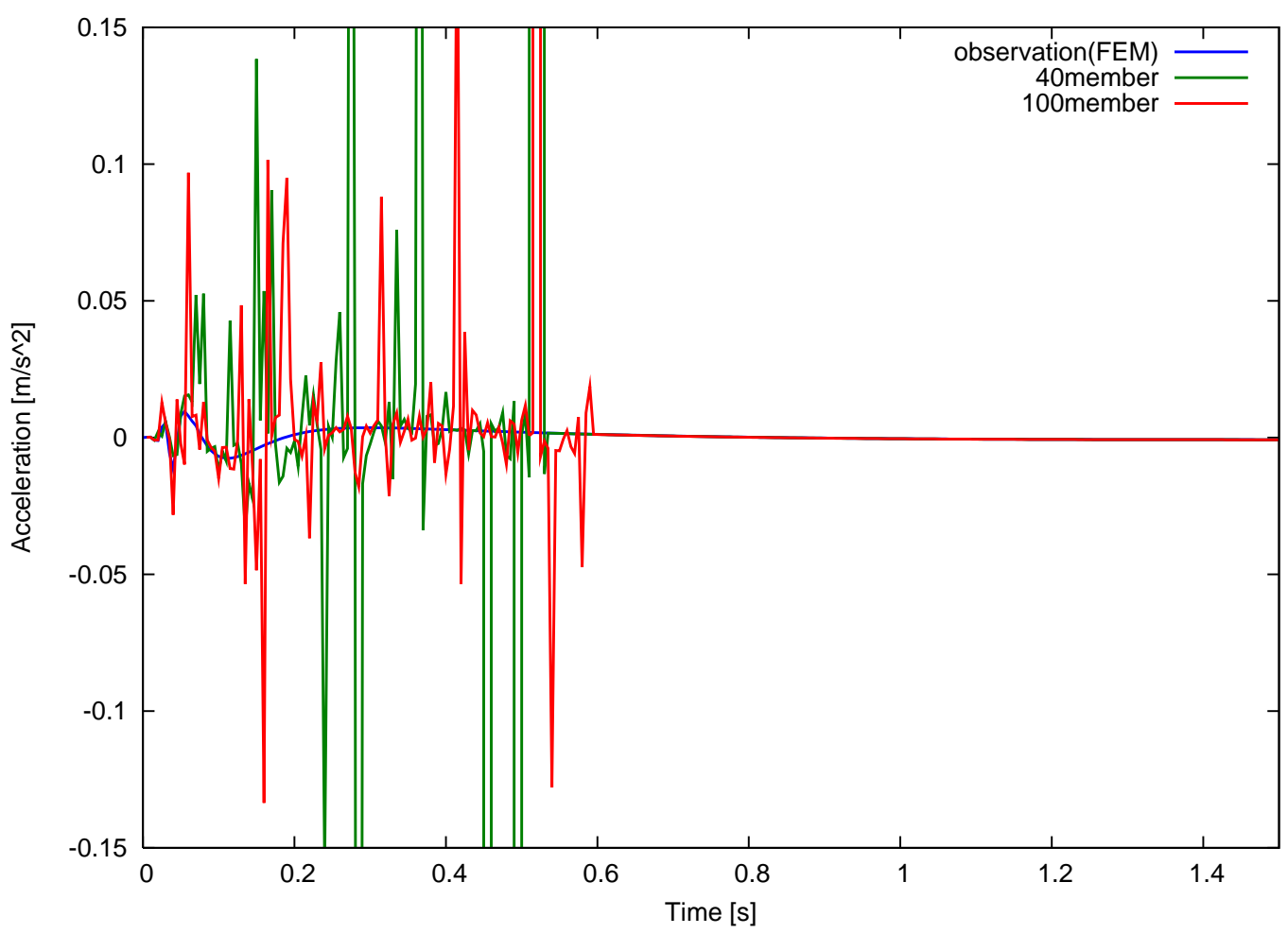

Figure 13. Y-acceleration at estimation point 


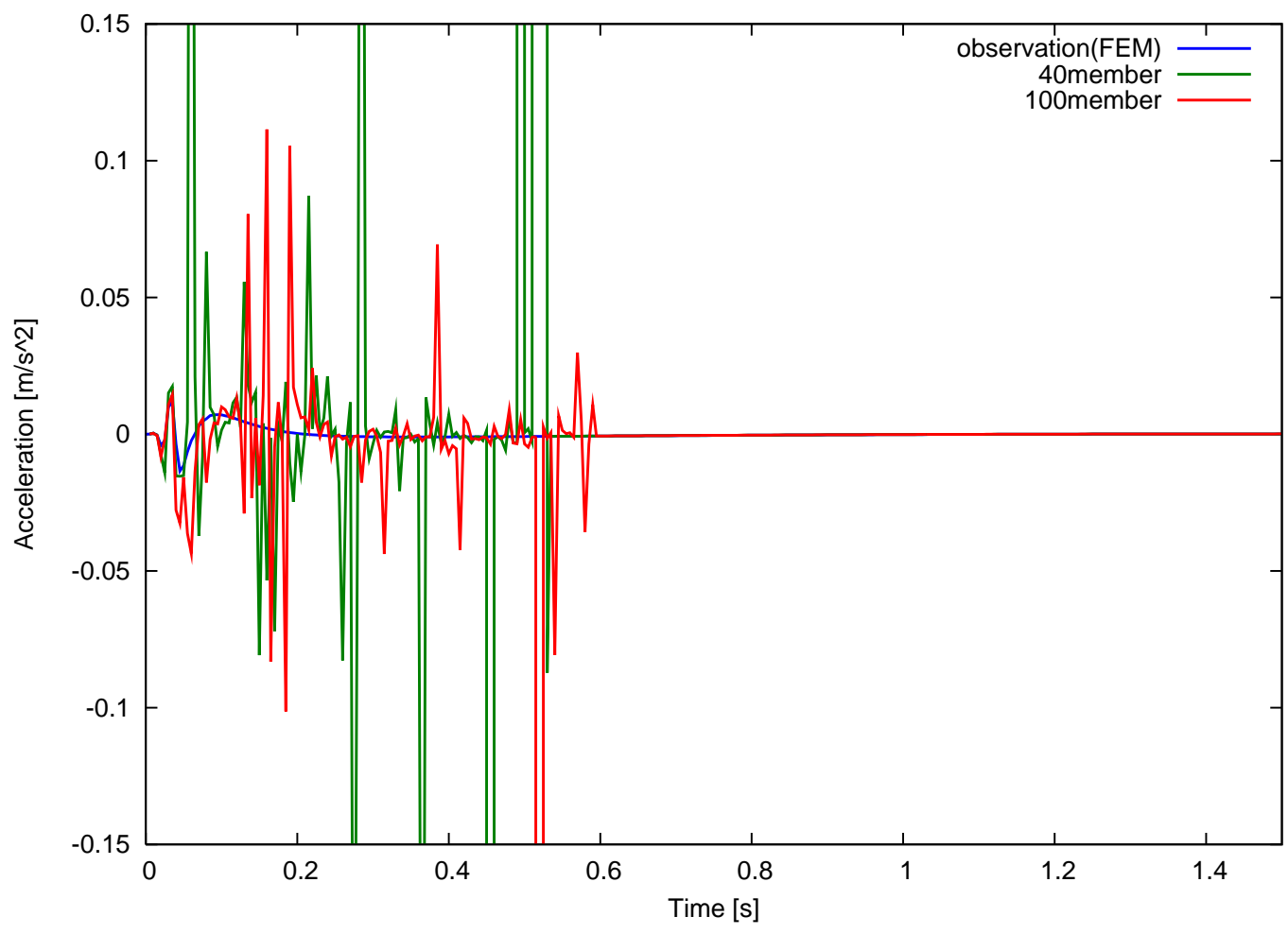

Figure 14. Z-acceleration at estimation point

\section{CONCLUSION}

It is found that the acceleration at estimation point can be estimated by the ensemble Kalman filter finite element method. The EnKF finite element method can be successfully applied to the cantilever beam and the Iwatayama tunnel model. The results of numerical experiments show that the estimated data of acceleration are well in agreement with the observed data. It is observed that, the accuracy of the EnKF increases when the number of ensemble members grows. As the future work, state value is estimated using actual measured data. In addition, the number of ensemble members should be increased.

\section{REFERENCES}

[1] S.Gillins, O.Barrero Mendoza, J.Chandrasekaer, B.L.R.De Moor, D. S. Bernstein, and A. Radley: "What Is the Ensemble Kalman Filter and How Well Does it Work?". Proceedings of the 2006 American Control Conference Minneapolis. Minnesota, USA, June 14-16, 2006.

[2] Geir Evensen: "The Ensemble Kalman Filter: theoretical formulation and practical implementation”. Ocean Dynamics(2003). 53: 343-367 DOI 10.1007/s10236-003-0036-9.

[3] A.Hommels and F.Molenkamp: "Inverse analysis of an embankment using the Ensemble Kalman Filter including heterogeneity of the soft soil". Numerical Methods in Geotechnical Engineering, H.Schweiger ed. pp.635-639, 2006.

[4] Y.Kato, M.Kawahara and N.Koizumi: "Kalman Filter Finite Element Method Applied to Dynamic Ground Motion". Int . J. Num. Analy. Meth. Geomech. vol.33, No.9, pp1135-1151, 2010. 